\title{
Glycine and D-serine improve the negative symptoms of schizophrenia
}

Tuominen HJ, Tiihonen J, Wahlbeck K. Glutamatergic drugs for schizophrenia: a systematic review and meta-analysis. Schizophr Res 2005;72:225-34.

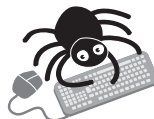

\section{Are glutamate receptor agonist drugs effective for people with schizophrenia?}

\section{METHODS}

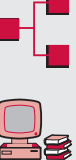

Design: Systematic review with meta-analysis.

Data sources: Studies were identified using the Cochrane schizophrenia group's trial register, BIOSIS Inside, CENTRAL CINAHL, EMBASE, MEDLINE, and PsycINFO plus handsearches and contact with investigators.

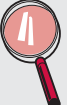

Study selection and analysis: Eligible studies were double blind randomised controlled trials (RCTs) of NMDA, AMPA, or kainate glutamate receptor agonist (glutamatergic) drugs in people with schizophrenia, with a trial duration of more than two weeks. Random and fixed effect models were used to carry out metoanalyses.

目事道

Outcomes: Global response (Clinical Global Impression scale (CGI); Global Assessment Scale (GAS)), negative symptoms (Positive and Negative Syndrome Scale (PANSS); Scale for Assessment of Negative Symptoms), and cognitive deficiencies (PANSS cognitive subscale).

\section{MAIN RESULTS}

Eighteen RCTs met inclusion criteria. The glutamatergic drugs investigated were D-cyloserine (7 RCTs), glycine ( 7 RCTs), and D-serine ( 3 RCTs); all were used in combination with antipyschotic medication. Glycine and D-serine significantly reduced the negative symptoms of schizophrenia, but there was no significant difference between D-cycloserine and placebo (glycine or D-serine $v$ placebo, $\mathrm{n}=132, \quad \mathrm{SMD}-0.66, \quad 95 \%$ CI -1.02 to $-0.29 ; \mathrm{p}=0.0004$; D-cycloserine $v$ placebo, $\mathrm{n}=119$ : SMD $-0.11,95 \%$ CI -0.48 to $0.25 ; \mathrm{p}=0.6$ ). There was no significant improvement in cognitive deficiencies with glycine or D-serine, and no consistent evidence of improvement in global response compared with placebo (cognitive deficiencies: glycine or D-serine $v$ control, $\mathrm{n}=80$, WMD -2.79 , $95 \%$ CI -6.17 to $0.60 ; \mathrm{p}=0.11$; global response: glycine $v$ placebo, dichotomous measures, $\mathrm{n}=53$, RR $0.48,95 \%$ CI 0.15 to $1.49 ; \mathrm{p}=0.2$; glycine $v$ placebo continuous measure (GAS), $\mathrm{n}=35$, WMD -3.87 , $95 \%$ CI -7.69 to $-0.05 ; \mathrm{p}=0.05$; D-serine $v$ placebo, continuous measure (CGI), $\mathrm{n}=49$, WMD -0.89 , 95\% CI -1.87 to +0.09 , $\mathrm{p}=0.07)$.

\section{CONCLUSIONS}

Glycine and D-serine improve negative symptoms, but not cognitive deficiencies or global response in people with schizophrenia in the short term.

\section{NOTES}

All of these studies investigated short term treatment ( $\leqslant 12$ weeks) with glutamatergic drugs and may not be applicable to long term treatment for people with schizophrenia.

For correspondence: Kristian Wahlbeck, National Research and Development Centre for Welfare and Health (STAKES), PO Box 220, FIN 00531 Helsinki, Finland; kristian.wahlbeck@stakes.fi

Sources of funding: The Wilhelm and Else Stockmann Foundation and special government funding (EVO).

\section{Commentary}

The enduring nature of cognitive and negative symptoms of schizophrenia represents an unmet therapeutic need. We recently suggested that the study of cognition and antipsychotics is not always driven by logic and that research into effective "pro-cognitive" or "antinegative" drug treatments must be guided by a better understanding of the biochemical mechanisms underlying cognitive processes and deficits.'

Many clinicians and researchers are currently considering augmentation strategies in an effort to improve the effectiveness of antipsychotics. Serious consideration has been given to agents such as D-cycloserine or ampakines that aim to enhance the function of the neurotransmitter glutamate in the brain. The hypothesis that a dysfunction of glutamatergic neurotransmission could play a key role in the deficits associated with schizophrenia is largely based on the discovery 40 years ago that drugs such as PCP and ketamine, which are now known to block NMDA-type glutamate receptors, were capable of inducing schizophrenia-like symptoms in humans. However, a further biological evidence supporting this theory is currently limited. The prospect of tinkering with the brain's most abundant neurotransmitter may seem a little risky to some neuroscientists. Nonetheless, drugs that act as co-agonists at the NMDA receptors show some promise.

The meta-analysis by Tuominen et al found that positive symptoms failed to respond to pro-glutamatergic medication. However, two of these agents (glycine and D-serine) were found to produce modest decreases in negative symptoms.

Since 2002, the search date of the Tuominen review, new trials of proglutamatergic drugs have been completed. An important multisite trial with 171 patients meeting the criteria for persistent negative symptoms was conducted using a random assignment to placebo, glycine, or D-cycloserine. ${ }^{2}$ The trial failed to confirm the efficacy of glycine or D-cycloserine to improve negative symptoms. The same team analysed a data set in this trial on cognition and event related potential (ERP). There was no significant effect on objective measures of cognition. ${ }^{3}$ The only anomaly on ERP was an increase of the P-50 amplitude on glycine, relative to placebo usually related to the sensory gating. ${ }^{4}$ Interestingly, the classes of antipsychotic medication that the patient received during the augmentation did not affect the findings. What can be concluded from such contradictory results? One would be tempted to say "formerly, we were indecisive, now we are not so sure".

Emmanuel Stip, MD, MSc, CSPQ

Centre de recherche Fernand Seguin and Department of Psychiatry, Université de Montréal, Canada Louis Eric Trudeau, PhD

Centre de recherche Fernand Seguin and Department of Pharmacology, Université de Montréal, Canada

1 Stip E, Chouinard S, Boulay L. On the trail of a cognitive enhancer for the treatment of schizophrenia. Prog Neuropsychoparmacol Biol Psychiatry 2005;29:219-32

2 Carpenter WT, Buchanan RW, Javitt DC, et al. Testing two efficacy hypotheses for the treatment of negative symptoms. Schizo Bull 2005;31:478.

3 Buchanan RW, Gold JM, Bilder R, et al. A summary of the FDA-NIMHMATRICS workshop on clinical trial design for neurocognitive drugs for schizophrenia. Schizo Bull 2005;31:5-19.

4 Summerfelt A, Francis $G$, Ball $P$, et al. The effect of glycine and Dcycloserine on sensory gating in patients with schizophrenia. Schizo Bull 2005;31:464.

An extended commentary by Emmanuel Stip and Louis Eric Trudeau on this article can be found at http://www.ebmentalhealth.com/supplemental 